\title{
ST-segment changes during tilt-table testing in postural tachycardia syndrome: are they relevant?
}

\author{
Frank I. Marcus ${ }^{1}$
}

Received: 6 December 2019 / Accepted: 9 December 2019 / Published online: 21 January 2020

c) Springer-Verlag GmbH Germany, part of Springer Nature 2020

Electrocardiographic (ECG) changes during head-up tilt have been previously reported in patients undergoing tilttable testing. For instance, in a large study including 150 patients with dissimilar diagnoses [including but not limited to orthostatic hypotension, vasovagal syncope, and postural tachycardia syndrome (POTS)], 135 had some ECG changes, most of them T-wave changes in inferior leads II, III and aVF and in anterolateral leads V3-V6. There was no evidence of myocardial ischemia in any of these patients. The relevance of these changes was unclear, but it was hypothesized that these alterations may be related to changes in the autonomic tone [1].

More recently, we reported that, among a sample of 180 patients, ST-segment changes during head-up tilt were found in $32 \%$ [2]. Of these, 28 (87.5\%) were women averaging 41 years of age. Since none of these women had a clinical history suggestive of coronary disease, it was postulated that these ST-segment changes were not due to ischemic heart disease. Still, these ECG abnormalities during head-up tilt remained largely unexplained.

In this issue of Clinical Autonomic Research, Vajapey and colleagues [3] confirm the relative high frequency of ST-segment changes during head-up tilt in patients with POTS. They studied an impressive sample of 255 patients with POTS who underwent both tilt-table testing and cardiac stress testing. Of these 255 patients, 45 had ST-segment changes or depression on head-up tilt. Forty-eight (91\%) were women, with an average age of 36 years. However, when evaluated with cardiac stress testing, none of these patients with ST-segment depression during head-up tilt had ischemic changes. This is a major contribution to the literature, thus clarifying in a systematic way that ECG changes

Frank I. Marcus

fmarcus@u.arizona.edu

1 Division of Cardiology, University of Arizona Medical Center, Sarver Heart Center, Room 5153, 1501 N Campbell Avenue, Tucson, AZ 85724-5037, USA in patients with POTS are not ischemic and are most likely benign.

However, we are still at a loss when interpreting these changes. It is good that they do not indicate coronary ischemic disease. This is not surprising, given that most patients with POTS are otherwise healthy young women, and therefore coronary ischemic changes would not be expected. Of note, most patients with POTS have hyperventilation during head-up tilt [4]. This is relevant because, more than half a century ago, T-wave inversions in two or more precordial leads were reported in 37 young healthy adults from a group of 350 subjects following brief hyperventilation [5]. These ECG changes were referred to as "juvenile pattern" and considered benign in nature. These 37 young adults were evaluated psychiatrically and were deemed to have underlying emotional issues, which interestingly, are also quite frequent in patients with POTS, many of whom have suicidal ideation [6].

In summary, although ST-wave changes during head-up tilt in patients with POTS appear benign in nature, their cause remains elusive. These might be related to the multifactorial comorbidities that frequently accompany patients with POTS including but not limited to hyperventilation or emotional disturbances. Additional studies on ECG changes upon head-up tilt taking into consideration these and other factors are warranted.

Funding None.

\section{Compliance with ethical standards}

Conflict of interest The author declares that they have no competing interests. 


\section{References}

1. Mayuga KA, Fouad-Tarazi F (2007) Dynamic changes in T wave amplitude during tilt table testing: correlation with outcomes. Ann Noninvasive Electrocardiol 12:246-250

2. Suryanarayana PG, Kandala J, Marcus FI (2017) High incidence of ST segment changes in women during tilt table testing. J Electrocardiol 50:884-888

3. Vajapey R, Hutt Centeno E, Van Iterson EH, Ahmed HM, Mayuga KA (2019) ST-segment changes during tilt table testing for postural tachycardia syndrome: correlation with exercise stress test results. Clin Auton Res. https://doi.org/10.1007/s10286-01900633-9
4. Stewart JM, Pianosi P, Shaban MA, Terilli C, Svistunova M, Visintainer P, Medow MS (2018) Postural hyperventilation as a cause of postural tachycardia syndrome: increased systemic vascular resistance and decreased cardiac output when upright in all postural tachycardia syndrome variants. J Am Heart Assoc 7(13): 0008854

5. Lewis WC, Siebecker KL Jr, Wasserburger RH (1956) The effect of hyperventilation on the normal adult electrocardiogram. Circulation 13(6):850-855

6. Pederson CL, Brook JB (2017) Health-related quality of life and suicide risk in postural tachycardia syndrome. Clin Auton Res 27(2):75-81 